\title{
Optimizing strategies to improve interprofessional practice for veterans, part I
}

\author{
This article was published in the following Dove Press journal: \\ Journal of Multidisciplinary Healthcare \\ 17 April 2014 \\ Number of times this article has been viewed
}

\author{
Shelley B Bhattacharya ${ }^{1-3}$ \\ Michelle I Rossi ${ }^{1,2}$ \\ Jennifer M Mentz'
}

'Geriatric Research Education and Clinical Center (GRECC), Veteran's Affairs Pittsburgh Healthcare System, 'University of Pittsburgh Medical Center, Pittsburgh, PA, USA; ${ }^{3}$ Albert Schweitzer Fellowship Program, Pittsburgh, PA, USA
Correspondence: Shelley B Bhattacharya University of Kansas Medical Center, 3599 Rainbow Blvd, Kansas City,

KS 66160, USA

$\mathrm{Tel}+$ I 9135880056

$\mathrm{Fax}+1913588$ I20I

Email sbhattacharya@kumc.edu

Michelle I Rossi

VA Pittsburgh Healthcare System GRECC

$\mathrm{Tel}+\mathrm{I} 4126922360$

Fax + I 4I23606159

Email rossimi@upmc.edu
Introduction: Interprofessional patient care is a well-recognized path that health care systems are striving toward. The Veteran's Affairs (VA) system initiated interprofessional practice (IPP) models with their Geriatric Evaluation and Management (GEM) programs. GEM programs incorporate a range of specialties, including but not limited to, medicine, nursing, social work, physical therapy and pharmacy, to collaboratively evaluate veterans. Despite being a valuable resource, they are now faced with significant cut-backs, including closures. The primary goal of this project was to assess how the GEM model could be optimized at the Pittsburgh, Pennsylvania VA to allow for the sustainability of this important IPP assessment. Part 1 of the study evaluated the IPP process using program, patient, and family surveys. Part 2 examined how well the geriatrician matched patients to specialists in the GEM model. This paper describes Part 1 of our study.

Methods: Three strategies were used: 1) a national GEM program survey; 2) a veteran/family satisfaction survey; and 3) an absentee assessment.

Results: Twenty-six of 92 programs responded to the GEM IPP survey. Six strategies were shared to optimize IPP models throughout the country. Of the 34 satisfaction surveys, $80 \%$ stated the GEM clinic was beneficial, 79\% stated their concerns were addressed, and $100 \%$ would recommend GEM to their friends. Of the 24 absentee assessments, the top three reasons for missing the appointments were transportation, medical illnesses, and not knowing/remembering about the appointment. Absentee rate diminished from $41 \%$ to $19 \%$ after instituting a reminder phone call policy.

Discussion: Maintaining the sustainability of IPP programs is crucial for the health of our veterans. This project uncovered tools to improve the GEM IPP model for our veterans that can be incorporated nationally. Despite the lengthy nature of IPP models, patients and families appreciated the thoroughness, requested transportation and food, and responded well to reminder phone calls. A keen eye on these issues and concomitant medical complexity needs to be observed when planning IPP models to ensure sustainability.

Keywords: interprofessional practice, veterans, geriatric evaluation and management

\section{Introduction}

Although interprofessional care of patients is only now gaining momentum among Accountable Care Organizations and health systems, the Veteran's Affairs (VA) Medical Center (VAMC) system has been incorporating this concept for decades. The goal of all outpatient VA Geriatric Evaluation and Management (GEM) programs is to provide comprehensive interprofessional geriatric assessments of veterans, targeting the medical, psychological, and social issues that affect the function and well-being of older veterans. GEM programs incorporate a range of specialties - including but 
not limited to, medicine, nursing, social work, physical therapy, and pharmacy - to collaboratively evaluate veterans. The GEM visit culminates in a cohesive patient care plan shared by all disciplines. Although they provide a valuable evaluation for veterans, GEM programs are faced with significant cut-backs, including closure, among VA hospitals nationally. The survival of these interprofessional programs within and outside the VA system is challenging due to the balance of other clinical commitments and the significant time commitments. Within the VA this type of interdisciplinary comprehensive geriatric assessment is mandated by the Veterans Millennium Health Care and Benefits Act (H.R.2116 ENR) ${ }^{1}$ of 1999. This Act includes a number of provisions for the care of older veterans in the VA health care system, including provisions for homecare services, palliative care, and respite care services, as well as geriatric assessments. ${ }^{1}$ Despite this mandate there is a struggle to maintain these programs because of competing budgetary demands. Each VA medical center must use its budget to fulfill all needed services and this balance of services is determined at each local facility. Additionally, programs may close because of attrition of staff - especially those with geriatric training - and the overall time commitment for this type of team-based intervention; low productivity because of missed patient appointments is another factor. ${ }^{2}$ The primary goal of this project was to systematically assess how the GEM model could be optimized to allow for the long-term sustainability of this important interprofessional practice (IPP) assessment. Part 1 of the study evaluated the IPP process using a national GEM forum, a patient/family satisfaction survey, and patient absentee data. Part 2 used a prediction tool to determine how well the GEM geriatrician matched patients to specialists in an IPP model.

Although the interprofessional approach to medical care has recently been gaining momentum in the health care arena, the VAMC recognized its importance many years ago. Formal geriatric assessment programs were first developed in Great Britain in the 1930s based on the work of Dr Marjory Warren and began in the United States in the 1970s. The VA had an early interest in the care of geriatric patients, which led to the establishment of Geriatric Research Education and Clinical Centers (GRECC). The first VA GEM was an inpatient model and opened in 1976 in Little Rock, Arkansas; subsequent outpatient GEM programs have since been developed and studied extensively. ${ }^{3,4}$

More recently, in 2010 a report by the "Study Group on Interprofessional Education and Collaborative Practice" of the World Health Organization affirmed "collaborative health education and practice as an effective and necessary approach to strengthening health care systems, both locally and globally." "In addition, the Institute of Medicine identified the ability "to work in interdisciplinary teams - cooperate, collaborate, communicate, and integrate care in teams to ensure that care is continuous and reliable" as one of three core competencies needed by health care professions. ${ }^{6}$

The important components of the GEM assessment include the following: 1) incorporating a team of physician and non-physician clinicians to assess each patient, discuss their findings, and develop an interprofessional care plan together; 2) targeting the most complicated patients to receive the GEM assessment; and 3) providing communication to the patient, family, and care providers to ensure that common goals are met. Currently, in the VA there are approximately 34 active inpatient GEM units and 49 active outpatient GEM programs. These programs vary in the way that they are designed. Some outpatient GEM clinics provide pure consultative service to primary care providers (PCPs), while others are integrated into geriatric primary care clinics. Inpatient GEM units are most often designated as Nursing Home Care Units (NHCU) but can also be acute or intermediate care units. The specific make-up of each GEM team also varies and is dependent on the local expertise and resources available at different VA medical centers. However, the underlying concept of comprehensive geriatric assessment using an interprofessional approach is constant.

The primary goal of our GEM IPP improvement project was to be accomplished with three strategies. First, we wanted to establish baseline data by surveying GEM's around the country, determine how they were structured (outpatient versus inpatient, consultative versus continuity care, one visit versus multiple visits) and what services they provided. We wanted to find successful strategies and challenges specific to each GEM, with the hopes of establishing a forum where constructive ideas could be freely exchanged between GEM programs to improve overall efficiency, patient care, and communication.

After collecting this data, the investigators chose the Pittsburgh, Pennsylvania GEM program as an intervention site. This program was chosen to target IPP improvement because it was the only outpatient VA GEM program where all patients saw all specialists on the initial evaluation; it was an outpatient, consultative, 3-day evaluation. The program had up to nine specialists working with the team: a geriatrician, nurse practitioner, psychologist, geriatric psychiatrist, physical therapist, occupational therapist, chaplain, pharmacist, and a social worker. Four or five of 
these specialists saw each patient on the first day of the evaluation and the remaining specialists saw each patient on the second day of the evaluation. These two evaluation days were generally 1 week apart (by patient and family preference). The GEM interprofessional team met to develop an interdisciplinary plan of care which was followed by a family meeting on the third day. At the family meeting, the patient and family were given a verbal and written summary of the individualized recommendations from the team of specialists. The primary care physician (PCP) also received a copy of the recommendations for follow-up, and the summary of recommendations was entered into the Computerized Patient Record System (CPRS). Patients did not continue their primary care at the Pittsburgh VA GEM Clinic; therefore, all care recommendations were directed to the PCP and/or subspecialists for follow-up. The patients returned to the GEM team annually for a condensed 1-day visit with one or two specialists to review progress from the initial evaluation. We hypothesized that the length and complexity of the Pittsburgh GEM evaluation would cause decreased patient and family satisfaction and lead to an increase in absenteeism. Therefore, interventions targeted to their model would likely help their IPP and also be helpful for other GEM's for utilization at their respective sites.

The second strategy was to assess veteran and family satisfaction at the Pittsburgh GEM program to determine the direction for improvement from the patient's perspective and to give the investigators guidance as to where changes could be made.

The third strategy was to conduct a patient absentee assessment to investigate the prime reasons patients didn't complete their scheduled appointment time at the Pittsburgh GEM. By understanding why patients hadn't come to their appointments, one could learn what strategies to employ to maintain patient volume.

By using data from the three sources above, our goal was to identify specific system tools to improve efficiency and satisfaction in the interprofessional GEM practice that could potentially be implemented throughout all VA Medical Centers.

As an important early model of IPP, GEM has been well studied and its benefits demonstrated in numerous past investigations. The investigative team reviewed eleven outpatient GEM studies closely when this project began. Mortality, care satisfaction, function, financial impact and utility of services were the major outcomes in these studies. Although each study had different primary endpoints and the inclusion criteria varied, all studies were in community dwelling adults and compared outcomes between a veteran GEM patient cohort and a non-GEM cohort.

The inclusion criteria for age differed slightly in the eleven studies; five recruited subjects over $70,{ }^{7-11}$ four over age $65,{ }^{12-15}$ and two over age $55 .{ }^{16,17}$ With regards to mortality, five displayed no difference in mortality rates between the two groups, ${ }^{7,10,12,15,16}$ two had decreased mortality, ${ }^{13,15}$ and four did not assess mortality. ${ }^{8,9,11,17}$ Since function is defined as the ability to perform the activities of daily living and is an important component of overall health, a common goal in geriatric care is to minimize the rate of functional decline. Seven studies had function as an outcome; five showed less decline in the GEM cohort, ${ }^{7,10,12-14}$ and two had the same rate of decline between the two groups. ${ }^{16,17}$ Although patients followed by an outpatient geriatric team showed less decline than their counterparts, it is important to note that the GEM design varied with each program; some received longitudinal care led by a geriatrician while others had one or two GEM visits with follow-up by a non-geriatrician.

Nine studies examined patient and provider care satisfaction using participant assessments and caregiver surveys as their tools. Eight showed an improvement in care satisfaction with the GEM cohort; ${ }^{7-9,11,14-17}$ and one found the satisfaction to be equivocal. ${ }^{13}$

The final outcome, cost analysis, was examined by four studies. One study found GEM programs to have decreased overall Medicare payments. ${ }^{7}$ Another study found a 34\% increase in expenditures in the first 8 months, followed by a $37 \%$ decrease in the last 8 months of the study. ${ }^{16}$ This is logical since the bulk of the consultations are performed within the first 2 months of a geriatric evaluation for most GEM programs. One study found equivocal expenditures between groups ${ }^{10}$ and one study found an increase in outpatient costs. ${ }^{11}$ The wide difference in financial outcomes is likely due to differing regional health care costs and different GEM protocols requiring subjects to have varying numbers of consultations. The challenge in comparing these eleven studies was that they each measured different outcomes and had subject populations with varying ages, levels of dementia, and physical function. Mortality and cost savings have not shown a clear advantage among the GEM cohort and further research needs to be performed to assess this relationship. Despite the varying protocols, the analysis does show that outpatients followed by a GEM team generally have improved care satisfaction and function. Both positive outcomes are important to quality care and need to be sustained when deciding on appropriate care for older adults. 


\section{Methods}

\section{Strategy I: National GEM review}

In 2004, according to the VA database, there were 92 GEM programs around the country. To elicit strategies that other GEM programs used to conduct their programs, all 92 programs were contacted three times by email in the fall of 2004 by a single investigator to respond to four questions asking 1) their location, 2) how their GEM clinic is structured, 3) their allocation of time between patients and specialists, and 4) their common problems and solutions. Participation was encouraged, but not mandatory, and there were no material incentives for participating.

\section{Strategy 2: assessing patient and family satisfaction}

Patient satisfaction was assessed from April 2004 to May 2005 by a patient and family quality improvement survey distributed on a rolling basis after each of the three Pittsburgh GEM IPP visits. The investigators and consultants, using a Likert format for ease of use, clarity and language, created the surveys. Nine questions were designed using a Likert scale or a yes/no multiple choice answer. The tenth question allowed for an area for written comments. The investigators attempted to survey everyone - surveys were given to each patient and one family member after each visit. Surveys were completed by the patients or their family members, in the office or at home, without GEM staff supervision. Participation was encouraged, but not mandatory, and there were no material incentives for participating. The only identifiable element was their age and their status as either the patient or family member. The ten-question survey inquired about how well the GEM clinic addressed their concerns, flow issues such as visit length, ease of appointment scheduling, their time with the specialists, and general feedback issues such as perceived problems and whether they would refer their friends to the GEM clinic. The survey that was distributed after the GEM team and family meeting also asked for feedback about the utility of the GEM recommendations and the team meeting. Upon completion, the patients and families were asked to anonymously return the surveys at a central location or by mail.

\section{Strategy 3: absentee assessment}

To assess reasons for missed appointments, a single investigator personally phoned each absent patient from April to November 2004 within 14 days of their scheduled appointment at the Pittsburgh GEM. If the patient was unavailable, the next of kin was asked to provide information. Their answers were tabulated under the following categories: transportation issues; patient forgot, family conflicts, or other. All data were analyzed using basic statistical methods.

\section{Results}

\section{Strategy I: National GEM review}

Of the 92 GEM programs that were contacted, 26 responded. Of the 26, nine no longer had a GEM program and only provided primary care services. Of the 17 remaining GEM programs, all were in the outpatient setting. Thirteen programs had a GEM clinic that operated under 3 half-days a week and four had a GEM clinic 3 or more half-days a week. For the initial GEM visit, seven of the programs had protocols that lasted under 2 hours, and ten had protocols lasting over 2 hours. The majority had each patient see the GEM physician, social worker, a trainee (geriatric fellow, internal medicine, or family medicine resident or medical student) and a nurse (registered nurse or nurse practitioner) on the first visit. Subsequent visits to see psychology, psychiatry, pharmacy, or physical therapy were based on the discretion of the initial GEM visit recommendations. Part 2 will investigate the successful prediction of these visits by the GEM geriatrician.

Although each outpatient clinic had its unique circumstances, consistently reported problems included a high absentee rate, poor compliance with GEM recommendations by the PCPs, and the lengthy duration of the visit. These challenges were solved as follows by a variety of interventions shared by the GEM programs (Table 1):

1. To reduce their absentee rate, many used a telephone reminder system 1-3 days before each scheduled visit to not only remind patients of their visit but to determine the reason for their visit and assess which health care specialists were needed to initially see the patient.

2. Another tactic to reduce absenteeism involved calling patients who didn't show up to their appointment to reschedule the visit.

3. The length of the visit was shortened at one site by writing all the consultation's notes at the end of the visit instead of during the visit.

4. To improve compliance with recommendations by the families and PCPs, some programs sent letters to the PCPs following the appointment and asked that a family member come with the patient to help ensure that the recommendations were being followed.

5. For overall system changes, one site placed suggestion cards in strategic places at the GEM clinic for patients 


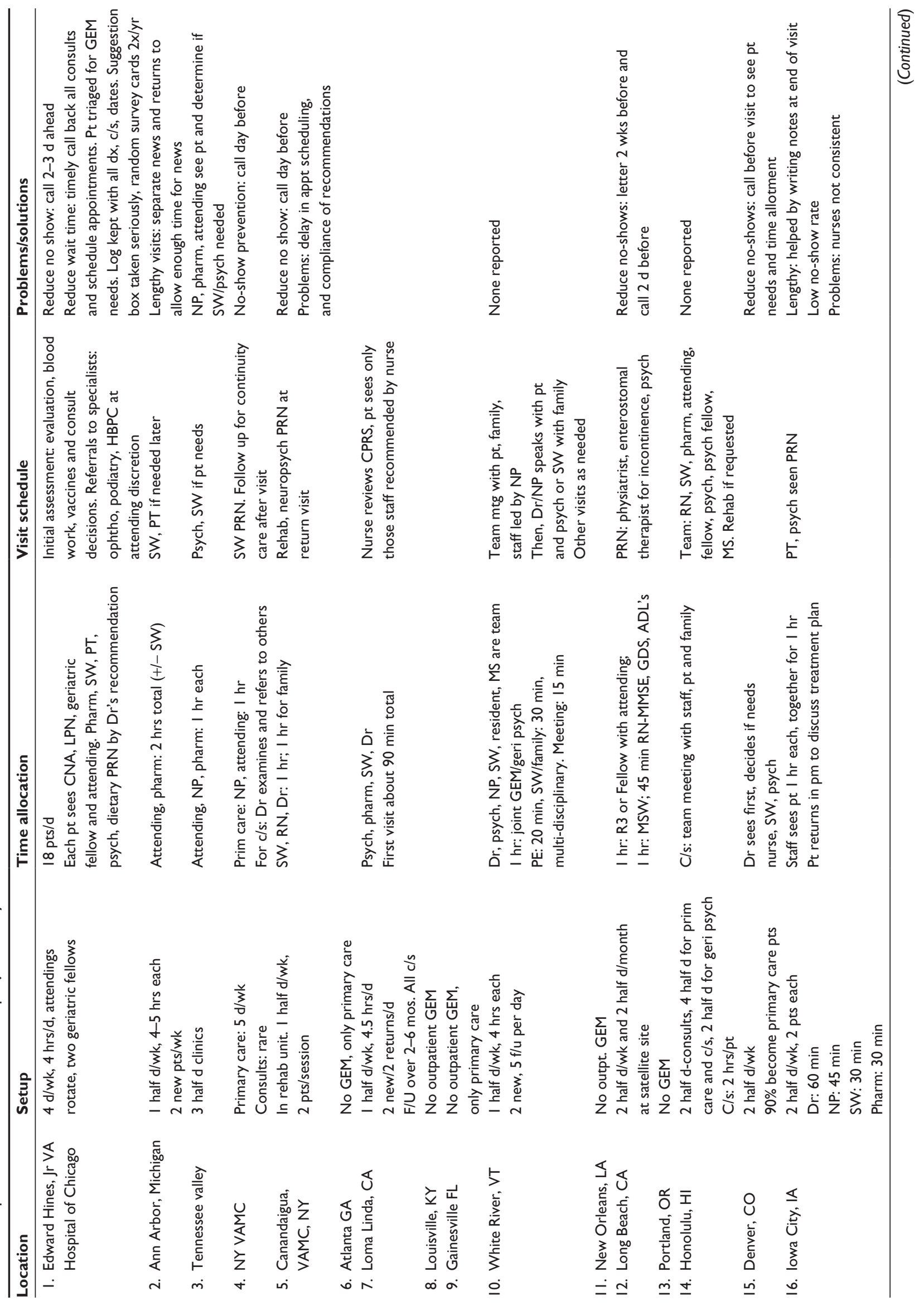




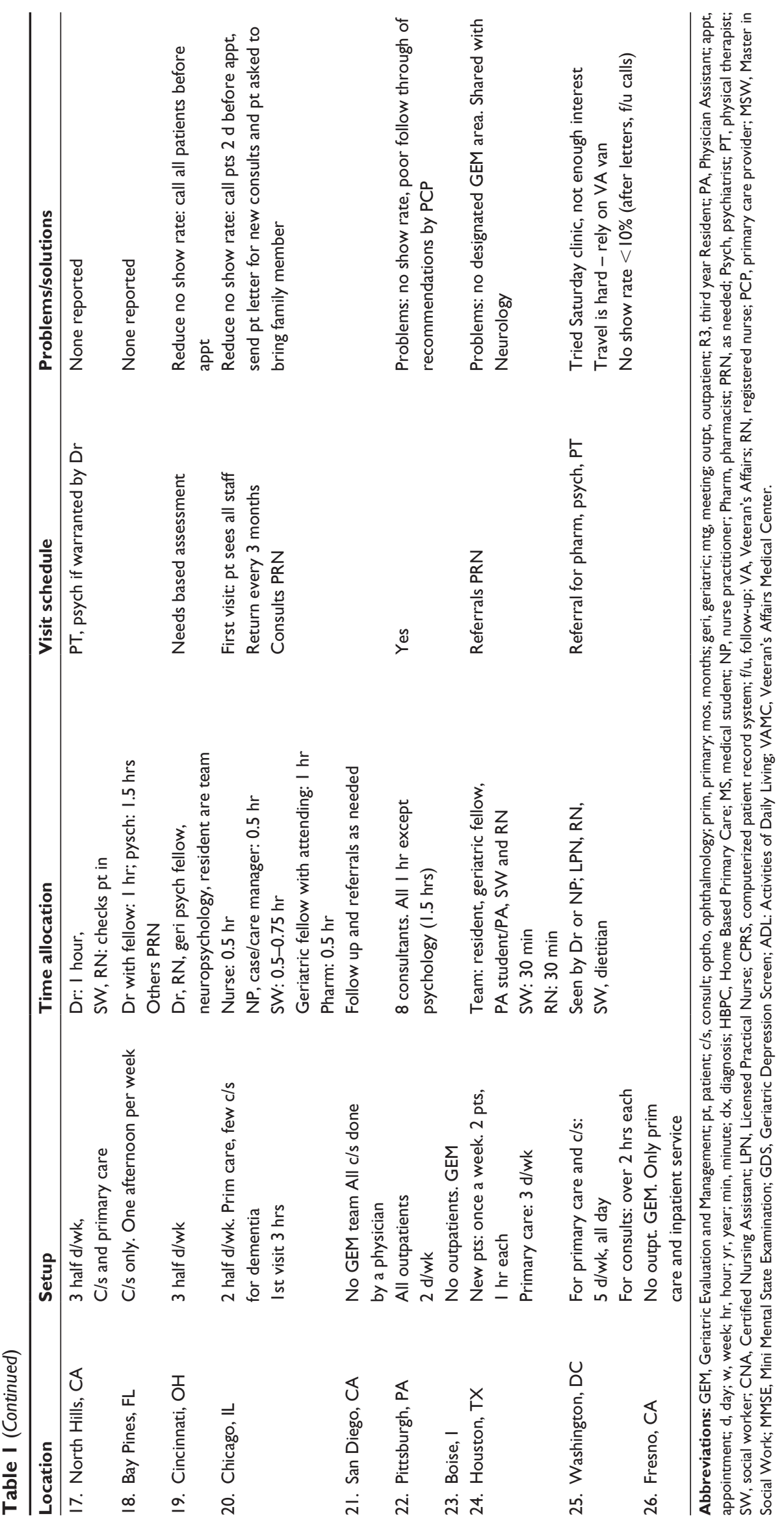


and families, and another mailed questionnaire cards to their patient panel twice a year.

6. One program reported transportation as a significant challenge and arranged for a VA van to coordinate transportation.

Overall, despite the low response rate, we understood the general structure, challenges, and solutions that many GEM programs had incorporated to maintain their success.

\section{Strategy 2: assessing patient and family satisfaction}

Between April 2004 and May 2005, 34 satisfaction survey forms were completed. Thirteen were completed after Day 1, 15 after Day 2, four after the team meeting, and two did not specify. Patients completed 23 of the forms (68\%) and family members completed 11 (32\%).

Missing values were not included in the analysis. The tabular results are shown in Table 2. Regarding the length of the visit, although three people stated the evaluation was too long, after the team meeting there were not any respondents who stated their GEM evaluation was too long. Ninety-three percent of the respondents did not have any problems during their visit; however, many wrote comments to improve the process. Comments included the need to have easier parking, a snack break, a lunch break longer than 30 minutes, allowing family members to participate with each specialist visit, and

Table 2 Survey results (n, 34)

\begin{tabular}{ll}
\hline & $\mathbf{n}(\%)$ \\
\hline The GEM clinic was & \\
Very beneficial & $7(2 \mathrm{I})$ \\
Somewhat beneficial & $20(59)$ \\
Not beneficial & $\mathrm{I}(3)$ \\
The GEM clinic addressed my concerns & \\
Very well & $14(4 \mathrm{I})$ \\
Somewhat well & $13(38)$ \\
Not at all & $2(6)$ \\
The visit length was & \\
Too long & $3(9)$ \\
Just right & $25(74)$ \\
Too short & $1(3)$ \\
Would you recommend GEM clinic to your friends? & \\
I would & $14(4 \mathrm{I})$ \\
I might & $13(38)$ \\
I would not & 0 \\
Difficulty getting an appointment? & \\
No & $25(74)$ \\
Yes & $2(6)$ \\
Don't know & $3(9)$ \\
\hline
\end{tabular}

Notes: Percentage sums in each category may not equal 100 due to rounding. Respondents may not equal 34 for each question as not all respondents answered all questions.

Abbreviation: GEM, Geriatric Evaluation and Management. fewer forms to complete. One respondent wrote that they had four of the same "memory tests" to complete. Forty-six percent found the visit to be "very enjoyable". The patients' PCPs referred $60 \%$ of the patients while family, friends, nurses and social workers referred the remainder. Nineteen percent of the respondents didn't know if their PCP knew they were getting evaluated.

After the team meeting, all felt that the GEM evaluation addressed their concerns "somewhat" to "very" well. None felt they saw too many specialists or that the evaluation was too long or too short. The team meeting was "pretty helpful" and the printed summary of recommendations was "pretty" to "very" helpful for all. One respondent stated that the recommendations were not easy to follow since they suggested that the respondent be actively involved with medication monitoring; however, the remainder found it easy to follow the recommendations. Overall, the GEM evaluation was "definitely" helpful and all respondents noted they would recommend the GEM clinic to others.

\section{Part III: absentee assessment}

During the 8-month study period, 170 patients were seen in the GEM clinic. After instituting the reminder phone call policy in August 2004, the absentee rate dropped from 41\% from April to July 2004 (41 no-shows out of 100 scheduled patients) to $19 \%$ from August to November 2004 (13 noshows out of 70 scheduled patients). Twenty-four patients could be reached by phone to inquire of their absence (Table 3). Family conflicts included issues in which the family could not accompany the patient, for example, insufficient lead-time to make the appointment date.

\section{Discussion}

Our project was designed to collect baseline data from various GEM programs around the country and implement the information to improve the sustainability of the IPP GEM clinic model in Pittsburgh, and potentially other GEM clinics nationwide. In particular, reducing the overall GEM visit duration, assessing patient and family satisfaction, and improving attendance rates were the domains chosen to investigate in the

Table 3 Reasons for absence (n, 24)

\begin{tabular}{lc}
\hline Transportation & $30 \%$ \\
Medical Illness & $27 \%$ \\
Didn't know & $17 \%$ \\
Family conflict & $9 \%$ \\
Not interested & $9 \%$ \\
Forgot & $4 \%$ \\
Out of town & $4 \%$ \\
\hline
\end{tabular}


Pittsburgh GEM program. The GEM forum was intended to begin communication between the GEM clinics to improve viability. Despite receiving responses from 26 of 92 clinics, valuable information was gained that could be shared and utilized by many GEMs immediately. Having feedback forms that are read and implemented, an automated phone reminder system, a VA van for transportation, and a phone triage system were tools that every GEM can consider implementing to maintain their viability. While some GEMs are part of larger VA medical centers with many resources and academic affiliations, others are in less resource-rich areas. These types of forums can be especially useful to share ideas and improve care in smaller centers or in those that are just getting started. Limited implementation of recommendations by the PCP's counterparts in the geriatric team was a consistent challenge noted by GEM clinics. This is a chronic issue noted in studies of consultant care. Improved implementation of recommendations has been shown to occur in settings where a geriatric team assumes primary care or there is a strong link between the geriatric team and the PCP. ${ }^{18}$ In general, this is likely due to limited communication between the two entities. Having the computerized medical record system is an asset within the VA system, yet the PCP team may not consistently access the GEM visit recommendations. In addition, without the GEM teams following up with the PCP teams, there are no consequences for not implementing the recommendations. The forum results are limited by response bias, since only $28 \%$ of the GEM programs responded.

The GEM patient and family satisfaction survey found that most patients and families were happy with the intervention despite its length. Limitations to this study include the possibility of reporting bias. Although all patients and families were contacted to complete the surveys, the 34 people who completed the surveys may have been those who had a primarily positive experience and were willing to complete the questionnaire; if this is the case, there was no feedback from those with negative experiences. In addition, the surveys were completed by respondents during different points of the GEM process and this could have changed their opinion of the process. Our numbers were small, which limits the power of this study. The concern that the 3-day assessment was too long was voiced by only a minority of patients. By the time they reached the team meeting, no one stated that the visit was too long or too short. This project did refute our theory that patients were unhappy with the length of the GEM intervention; overall, patients and families reported a positive experience and found the evaluation to be the right length.
Regarding the absentee data, the high prevalence of dementia in our clinic population likely contributed to the incidence of patients forgetting or being unaware of their appointments despite written and telephone reminders. As expected, transportation issues were a frequent cause of absence. To compensate, when GEM staff suspected patients with significant dementia had poor social support and transportation difficulties, they were often referred to Home Based Primary Care (HBPC) for continuing care and case management in their home. A high percentage missed their appointment due to medical illness, emphasizing the degree of medically complex patients referred to the GEM clinic. Our absentee rate improved significantly (by 22\%) by incorporating the phone reminder technique shared by the GEM forum; this may be reflected in the survey results showing that $17 \%$ missed their appointment due to "not knowing about it" and 4\% missed their appointment due to "forgetfulness". It is important to note that the improvement may have also been due to multiple external factors unrelated to the phone call reminder, for instance, weather, vacation, or time of year. The next steps for the Pittsburgh GEM are to continue implementing the reminder phone system, improve communication to the PCP teams, and maintain the current protocol of 2 days of interviewing and 1 day of family/team discussion.

The need to continue to find ways to care for our older adults was reiterated in the April 2008 Institute of Medicine report, "Retooling for an Aging America: Building the Health Care Workforce." This report concluded that the future workforce "will be woefully inadequate in its capacity to meet the large demand for health services for older adults if current patterns of care and of the training of providers continue." The number of older Americans will nearly double to 70 million by 2030 , the report notes, at which point the youngest of the baby boomers will have reached retirement age. ${ }^{19}$

It is critical that GEM programs serve older veterans in the most effective way possible. Interprofessional GEM programs can improve the care of older adults but also require extra time and personnel to assess older veterans. With diminishing budgets, support for these programs is at risk. With the escalating geriatric cohort and the relative paucity of geriatricians, outpatient consultative interprofessional geriatric evaluations are a vital tool that has the potential to not only help PCPs better manage their geriatric patients, but also to teach them about management of geriatric syndromes. Multiple studies have shown the benefits of an outpatient interprofessional GEM program. Morishita et al found a $9 \%$ increase in patient mean satisfaction with GEM 
programs when compared to usual outpatient care, as well as a concomitant rise in satisfaction from primary physicians of GEM recipients. ${ }^{9}$ Toseland et al found that although cost reduction may not be achieved with GEM evaluations, the multidisciplinary aspect of outpatient GEMs are "an efficient and effective means of improving the care and well-being of frail elders." 16

Further research needs to be done to determine cost effective methods to maintain comprehensive geriatric evaluation programs at VA and non-VA facilities. With the geriatric sector approaching $20 \%$ by 2020 and with health care reform on the current legislative agenda, this service will need to be offered in a financially sound manner. By incorporating the recommendations described in this paper and with further research, outpatient GEMs can be a costeffective geriatric assessment tool appealing to the patient, provider, and payer.

\section{Acknowledgments}

The primary author wishes to acknowledge coauthors Michelle I Rossi and Jennifer M Mentz for their unending support of this project - both were crucial to its success. The Albert Schweitzer Fellowship Program and the University of Pittsburgh Medical Center provided the time to conduct this project; the VA Hospital in Pittsburgh provided the patient panels. Lastly, the authors would like to sincerely thank the patients and their families for their patience in completing the forms, answering telephone calls, and providing their feedback. It is for them that we strive to improve geriatric care. Dr Bhattacharya received funding from the University of Pittsburgh Medical Center, the Veteran's Affairs Pittsburgh Healthcare System, and the Albert Schweitzer Fellowship Program.

\section{Author contributions}

The authors had complete independence in the study concept and design, acquisition of subjects and/or data, analysis and interpretation of data, and preparation of manuscript. The sponsors did not have any role in the study design, methods, subject recruitment, data collections, analysis or preparation of the manuscript.

\section{Disclosure}

The authors report no conflicts of interest in this work.

\section{References}

1. Veterans Millenium Health Care and Benefits Act [webpage on the internet]. New York: About.com; 2013. Available from: http://usgovinfo.about. com/library/weekly/blvabill2000.htm. Accessed September 30, 2013.

2. Maly RC, Leake B, Frank JC, DiMatteo MR, Reuben DB. Implementation of consultative geriatric recommendations: the role of patient-primary care physician concordance. $J$ Am Geriatr Soc. 2002;50(8):1372-1380.

3. Rubenstein LZ, Joseph T. Freeman award lecture: comprehensive geriatric assessment: from miracle to reality. J Gerontol A Biol Sci Med Sci. 2004;59(5):473-477.

4. Geriatric Evaluation and Management Procedures. Veterans Health Administration (VHA) Handbook 1140.1, February 2, 2005. Washington: US Department of Veterans Affairs; 2005.

5. World Health Organization, Deptartment of Human Resources for Health. Framework for Action on Interprofessional Education and Collaborative Practice. Geneva, Switzerland: World Health Organization; 2010.

6. Institute of Medicine, Committee on the Health Professions Education Summit, Board on Health Care Services. Available from: http://www. iom.edu/Reports/2008/Retooling-for-an-aging-America-Building-theHealth-Care-Workforce.aspx. Accessed September 30, 2013.

7. Boult C, Boult LB, Morishita L, Dowd B, Kane RL, Urdangarin CF. A Randomized clinical trial of outpatient geriatric evaluation and management. J Am Geri Soc. 2001;49(4):351-359.

8. Weuve JL, Boult C, Morishita L. The effects of outpatient geriatric evaluation and management on caregiver burden. Gerontologist. 2000;40(4):429-436.

9. Morishita L, Boult C, Boult L, Smith S, Pacala JT. Satisfaction with outpatient geriatric evaluation and management (GEM). Gerontologist. 1998;38(3):303-308.

10. Rubin CD, Sizemore MT, Loftis PA, de Mola NL. A randomized, controlled trial of outpatient geriatric evaluation and management in a large public hospital. J Am Geri Soc. 1993;41(10):1023-1028.

11. Engelhardt JB, Toseland RW, O'Donnel JC, Richie JT, Jue D, Banks S. The effectiveness and efficiency of outpatient geriatric evaluation and management, J Am Geri Soc. 1996;44(7):847-856.

12. Burns R, Nichols LO, Martindale-Adams J, Graney MJ. Interdisciplinary geriatric primary care evaluation and management: Two-year outcomes. J Am Geri Soc. 2000;48(1):8-13.

13. Reuben DB, Frank JC, Hirsch SH, McGuigan KA, Maly RC. A randomized clinical trial of outpatient comprehensive geriatric assessment coupled with an intervention to increase adherence to recommendations. J Am Geri Soc. 1999;47(3):269-276.

14. Silverman M, Musa D, Martin DC, Lave JR, Adams J, Ricci EM. Evaluation of outpatient geriatric assessment: A randomized multi-site trial. J Am Geri Soc. 1995;43(7):733-740.

15. Boult C, Boult L, Murphy C, Ebbitt B, Luptak M, Kane RL. A controlled trial of outpatient geriatric evaluation and management. $J$ of Am Geri Soc. 1994;42(5):465-470.

16. Toseland RW, O’Donnell JC, Engelhardt JB, Richie J, Jue D, Banks SM. Outpatient geriatric evaluation and management: Is there an investment effect? Gerontologist. 1997;37(3):324-332.

17. Selim AJ, Berlowitz DR, Fincke G, et al. The health status of elderly veteran enrollees in the Veterans Health Administration. J Am Geri Soc. 2004;52(8):1271-1276.

18. Stuck AE, Siu AL, Wieland D, et al. Comprehensive geriatric assessment: a meta-analysis of controlled trials. Lancet. 1993;342:1032-6.

19. Committee on the Future Health Care Workforce for Older Americans. Retooling for an Aging America: Building the Health Care Workforce. Washington: Institute of Medicine; 2008. 


\section{Publish your work in this journal}

The Journal of Multidisciplinary Healthcare is an international, peerreviewed open-access journal that aims to represent and publish research in healthcare areas delivered by practitioners of different disciplines. This includes studies and reviews conducted by multidisciplinary teams as well as research which evaluates the results or conduct of such teams or

healthcare processes in general. The journal covers a wide range of areas and welcomes submission from practitioners at all levels, from all over the world. The manuscript management system is completely online and includes a very quick and fair peer-review system. Visit http://www.dovepress.com/testimonials.php to read real quotes from published authors.

Submit your manuscript here: http://www.dovepress.com/journal-of-multidisciplinary-healthcare-journal 